\title{
Run Regular, Age Slower, Be Neuroplastic
}

\author{
Farid Khan ${ }^{1, *}$ \\ ${ }^{1}$ Research Associate Neurology, the Aga Khan University Hospital Karachi, Pakistan \\ "Corresponding author: Farid Khan, B11, Pak Tameer Plaza, Gulshan e Iqbal, Block 14, Karachi. Pakistan 75300, E-mail: drfaridkhan@hotmail.com
}

Received 2016 November 09; Revised 2017 February 13; Accepted 2017 February 21.

\begin{abstract}
Background: Aging is interpreted as a physiological process, which appears in the form of progressive waning in organ function. Oxidative stress is considered as one of the central mechanisms of aging, which can be controlled by life style modification.

Objectives: We aimed to understand the impact of exercise on aging and mental health in light of the current research.

Methods: Article search was performed using PubMed search engine.

Conclusions: Exercise is the key to boost anti-oxidant pathways to slow aging and thus improve brain function and mental health.
\end{abstract}

Keywords: Exercise, Brain, Aging, Brain, Aging, Brain, Neuroplasticity Mental Health

\section{Brief Review}

Aging has been the debate for a while now, above all, the various factors responsible for it. Based on the irrefutable evidence of research studies and experimentation, aging is interpreted as a physiological process which appears in the form of progressive waning in organ function. Decline in organ function occurs at cellular and molecular levels. Biochemical molecules which are responsible for cellular processes become blunt, rigid, and less active to perform the labor assigned to them thwarting the cells to work normally like they used to. It is important to note that all of these phenomena do not occur methodically or simultaneously. For instance, a cell might lose a particular function but other cellular pathways work routinely. Aging occurs in each cell of every organ resulting in accumulative decline in bodily functions. Interestingly, this complex physiological process is observed outwardly, and may appear as the scheme of a hidden artist changing the shape of life.

Oxidative stress is considered as one of the important thoughts behind aging for the readily explanation of how waning phenomenon takes place (1). Free radicals produced inside the cell blemish biochemical molecules and proteins. If endogenous anti-oxidant counterbalance system is nonexistent or not working by the book, the free radicals will induce oxidative stress on cellular molecules theoretically and result in aging and related diseases. Injured mitochondria also produce reactive oxygen species (ROS) augmenting the damage to cellular molecules and function.

\section{Physical Fitness: Mechanism to Slow the Speed of Ag- ing}

One enormous benefit gained from the oxidative stress theory is that delay in aging can be achieved by increasing antioxidant defenses, concurrently delaying age-related diseases. Thanks to accelerated medical research, we currently have plentiful evidence that exercise improves antioxidant defenses by elevating antioxidant enzyme production and as a result, aging process slows down (1).

Physical activity such as exercise has been observed to reduce obesity, cardiovascular diseases, diabetes, stroke and other diseases that come under the pennant of metabolic syndrome. Current evidence also shows that exercise has substantial impact on brain function and mental health. Regular exercise has been found to enhance antioxidant systems in the neural tissue which result in slowing down brain aging and delaying onset of age-related brain disorders. One of the many age-related brain disorders is cognitive decline which is slowed or to some extent hindered by regular exercise. The doldrums of mind commonly called mood disorders also benefit from exercise and thus present as improved mental health.

\section{Rewire Your Brain by Doing Regular Exercise}

Brain matter, once believed a fixed tissue with almost nonexistent ability to repair or regenerate, is considered as the most dynamic organ now. Contrary to the previous myths, neuroplasticity and neurogenesis are the hallmarks of complex processes which enable the neural tissue to repair, modify, and resist damage. Experts wonder

Copyright (c) 2017, Tehran University of Medical Sciences. This is an open-access article distributed under the terms of the Creative Commons Attribution-NonCommercial 4.0 International License (http://creativecommons.org/licenses/by-nc/4.0/) which permits copy and redistribute the material just in noncommercial usages, provided the original work is properly cited. 
to contrive the ways of allowing and enhancing neuroplasticity and neurogenesis to increase the capacity of neural tissue to repair, adapt, and resist tiredness and injury.

In simple words, neuroplasticity can be described as the process of increasing inter-neuronal connections and resisting decrease in the same. In other words, a wired brain should have the ability to rewire connections, rewrite new neural scripts, and uphold the old ones. Cognitive function and healthiness of neural tissue are roughly the output of processes inside the neurons and interneuronal connections. Degree of complexity of the same should cause variation in the level of cognitive function. One central process of neuroplasticity is synaptic plasticity indicative of the number, structure, and functional facets of synapses. Regular exercise and fitness activity are thought to enhance both synthesis and release of circulatory factors which in turn enhance neuroplasticity and complexity of synapses. In animal studies, runner rats were observed with intensified synapses when compared to sedentary rats.

Similar to neuroplasticity, neurogenesis also has an imperative role in preserving brain function and improving cognition (2). Animal studies demonstrated enhanced neurogenesis in hippocampus amongst running rats, while the decline in the same ability was discovered to be associated with aging and appearance of mood disorders.

Research studies suggest the improved blood flow towards the brain simultaneously carrying neurotropic and other circulatory factors stimulated by exercise $(3,4)$. Increased amount of neurotrophins such as brain-derived neurotrophic factor (BDNF) which is associated with exercise has been found in both animal and human studies. Increased levels of BDNF allow neural tissue growth, survival and synaptic plasticity. Functional capacity of the brain maxed out after regular sessions of exercise. Participants involved in regular exercise had improved cognition, mood, concentration, and memory preservation.

There is ample epidemiological evidence for the close link between mental health and physical activity. Kramer et al. observed the direct relationship of exercise with cognitive function and brain mass in older adults (5). Retirees who continued exercise had superior cognitive function when compared to a group of inactive retirees. The idea cannot be overlooked due to the overall positive impact not only on mental health but also on the entire organ systems.

The most recent observation by Fahimi et al. at Stanford University School of Medicine showed that physical activity not only caused elevated levels of BDNF mRNA but also altered astrocytes morphology and orientation of their projections in regularly exercising mice (6). Since glial cells are non-neural in origin, the effect of physical activity on glial cells further proved in their observation that exercise had an overall positive impact on organ systems other than affecting neural tissue. Strengthening of astrocytes should potentially counter aging and neurodegenerative disorders.

\section{Our Ancestors Never Ordered Food Online but Were Out to Hunt}

Humans, to a great extent, are efficient endurance runners. Evolutionary scientists are of the opinion that high endurance capacity might be the evolutionary benefit humans inherited from ancestors who lived an al fresco life $(7,8)$. Unlike other mammals, increased anaerobic metabolism enabled humans to run for extended period of time and distance. Admittedly, in evolutionary history we observe healthy and long-aged humans. Another explanation is that human bodies have evolved to be dynamic to keep its functionality in normal state. Sedentary life style is considered as the abnormal state of the human body affecting almost every organ which brings us back to the oxidative stress theory.

In light of the previous discussion, exercise and regular physical activity are extremely important for humans to keep overall functionality in the normal state. Physical activity is the cheapest and easily available natural way to delay aging and improve mental health. Regular exercise should be accepted as the need of the body, not just a leisure activity to achieve endorphin boost. Humans have to change this behavior towards exercise and have a handle on what the needs of their physiology are.

\section{Footnotes}

Funding/Support: It is stated that no funding was received in any form for completing this brief review.

Conflicts of Interest: The author declares no potential conflicts of interest with respect to the authorship or publication of this article.

\section{References}

1. Garatachea N, Pareja-Galeano H, Sanchis-Gomar F, Santos-Lozano A, Fiuza-Luces C, Moran M, et al. Exercise attenuates the major hallmarks of aging. Rejuvenation Res. 2015;18(1):57-89. doi: 10.1089/rej.2014.1623. [PubMed: 25431878].

2. van Praag H. Neurogenesis and exercise: past and future directions. Neuromolecular Med. 2008;10(2):128-40. doi: 10.1007/s12017-008-8028-z. [PubMed: 18286389].

3. van Praag $\mathrm{H}$, Fleshner M, Schwartz MW, Mattson MP. Exercise, energy intake, glucose homeostasis, and the brain. $J$ Neurosci. 2014;34(46):15139-49. doi: 10.1523/JNEUROSCI.2814-14.2014. [PubMed: 25392482]. 
4. Voss MW, Vivar C, Kramer AF, van Praag H. Bridging animal and human models of exercise-induced brain plasticity. Trends Cogn Sci. 2013;17(10):525-44. doi: 10.1016/j.tics.2013.08.001. [PubMed: 24029446].

5. Kramer AF, Erickson KI, Colcombe SJ. Exercise, cognition, and the aging brain. J Appl Physiol (1985). 2006;101(4):1237-42. doi: 10.1152/japplphysiol.00500.2006. [PubMed:16778001].

6. Fahimi A, Baktir MA, Moghadam S, Mojabi FS, Sumanth K, McNerney MW, et al. Physical exercise induces structural alterations in the hip- pocampal astrocytes: exploring the role of BDNF-TrkB signaling. Brain Structure Function. 2016 doi:10.1007/s00429-016-1308-8.

7. Bramble DM, Lieberman DE. Endurance running and the evolution of Homo. Nature. 2004;432(7015):345-52. doi: 10.1038/nature03052. [PubMed: 15549097].

8. Mattson MP. Lifelong brain health is a lifelong challenge: From evolutionary principles to empirical evidence. Ageing Res Rev. 2015;20:37-45. doi:10.1016/j.arr.2014.12.011. 